\title{
Rastreo de hipercolesterolemia en niños y adolescentes
}

\author{
Lipid screening in childhood and adolescence
}

Lozano P. JAMA. 2016;316(6):634-644.

\section{Objetivo}

Sintetizar la evidencia sobre los beneficios y los daños de la detección de dislipidemia multifactorial (la forma más frecuente de hipercolesterolemia primaria) en niños y adolescentes (NyA) asintomáticos.

\section{Fuentes de datos}

Se buscaron en MEDLINE PubMed y el Registro Cochrane Central de Ensayos Controlados, estudios publicados entre el 1 de enero de 2005 y el 2 de junio de 2015. Se revisaron además todos los estudios incluidos en un informe de la Fuerza de Tareas Preventivas de los EE.UU. (sigla en inglés USPSTF) del año 2007 sobre este mismo tópico y las listas de referencias de estudios relevantes y en curso.

\section{Extracción y síntesis de datos}

Dos investigadores revisaron de forma independiente resúmenes y artículos de texto completo y extrajeron los datos en tablas de evidencia. Se desarrollaron ocho preguntas claves sobre el efecto del rastreo, tratamiento y pronóstico. Los resultados en salud del adulto incluyeron infarto de miocardio (IAM) y accidente cerebrovascular isquémico (ACV). Los resultados intermedios incluyeron concentraciones de lípidos (Colesterol Total [CT], LDLC, HDL-C, colesterol no HDL y triglicéridos) y marcadores de ateroesclerosis (espesor miointimal carotídeo, score de calcio y hallazgos de autopsias)

\section{Resultados}

Los principales resultados encontrados en cuanto a rastreo, tratamiento y pronóstico se describen en la Tabla 1.

No se encontraron estudios para responder las siguientes preguntas:

Sobre el rastreo: ¿Disminuye la incidencia de IAM o ACV en la edad adulta? ¿Mejora los resultados intermedios (concentraciones de lípidos o progresión de la aterosclerosis) en la infancia y la adolescencia? ¿Cuáles son sus daños?

Sobre intervenciones multifactoriales con modificaciones en el estilo de vida y/o medicamentos hipolipemiantes: ¿Disminuyen la incidencia de IAM y /o ACV en la edad adulta?

Tabla 1. Resumen de la evidencia en relación a las preguntas clave.

\begin{tabular}{|c|c|c|c|c|}
\hline Pregunta clave & $\begin{array}{l}\text { Cantidad de } \\
\text { estudios, diseño } \\
\text { y pacientes } \\
\text { involucrados ( } n \text { ) }\end{array}$ & Resultados & Limitaciones & Calidad \\
\hline ¿Cuál es su rendimiento diagnóstico? & $\begin{array}{l}4 \text { cortes } \\
\text { transversales } \\
(\mathrm{n}=78.792)\end{array}$ & $\begin{array}{l}\text { Rendimiento diagnóstico: } 5,8 \% \\
\text { (utilizando prueba confirmatoria) y de } \\
4,8-12,3 \% \text { con una sola determinación }\end{array}$ & $\begin{array}{c}\text { Población blanca } \\
\text { Prueba confirmatoria de } \\
\text { dislipidemia en un solo estudio }\end{array}$ & Buena \\
\hline $\begin{array}{l}\text { ¿Mejora los resultados intermedios (concentraciones de } \\
\text { lípidos, progresión de la aterosclerosis) en la infancia y la } \\
\text { adolescencia? }\end{array}$ & $\begin{array}{l}2 \text { ECCA } \\
(n=695)\end{array}$ & $\begin{array}{l}\text { Disminución de CT (-6.1 mg\%; IC95\% } \\
-9,1 \text { a -3,2) y en LDL en grupo } \\
\text { intervención (dieta intensiva) al año y } 3 \\
\text { años de seguimiento } \\
\text { La suplementación con linaza no } \\
\text { produjo ningún efecto sobre los lípidos } \\
\quad \text { a las } 4 \text { semanas }\end{array}$ & $\begin{array}{l}\text { Puntos de corte inferiores a los } \\
\text { reportados en la literatura }\end{array}$ & Buena \\
\hline $\begin{array}{l}\text { ¿Cuáles son los daños de las intervenciones multifactoriales } \\
\text { con modificaciones en el estilo de vida y/o medicamentos } \\
\text { hipolipemiantes? }\end{array}$ & $\begin{array}{l}1 \text { ECCA } \\
(n=663)\end{array}$ & $\begin{array}{l}\text { No se reportaron daños durante el } \\
\text { seguimiento en antropometría, } \\
\text { laboratorio, maduración o psicosociales }\end{array}$ & $\begin{array}{l}\text { Puntos de corte } \\
\text { inferiores a los reportados } \\
\text { en la literatura }\end{array}$ & Buena \\
\hline $\begin{array}{l}\text { ¿Cuál es la asociación entre los resultados intermedios en la } \\
\text { infancia y adolescencia y la incidencia futura de infarto de } \\
\text { miocardio y eventos cerebrovasculares en adultos? }\end{array}$ & $\begin{array}{c}\text { Análisis de datos } \\
\text { extraídos } \\
\text { del estudio } \\
\text { NHANES } \\
(n=9.245)\end{array}$ & $\begin{array}{l}\text { Las principales causas de muerte entre } \\
\text { los } 12 \text { y } 19 \text { años de edad fueron } \\
\text { lesiones involuntarias o autoinfligidas, } \\
\text { causas circulatorias y cáncer. } \\
\text { No se encontró asociación entre niveles } \\
\text { elevados de colesterol y muerte }\end{array}$ & $\begin{array}{l}\text { Población de } 12 \text { a } 39 \text { años } \\
\text { Resultados no reportados por } \\
\text { separado }\end{array}$ & Buena \\
\hline
\end{tabular}

ECCA: Ensayo clínico controlado aleatorizado; NHANES: National Health and Nutrition Examination Survey; CT: Colesterol Total

\section{Conclusiones}

El rendimiento diagnóstico de la detección de lípidos varía según la edad y el índice de masa corporal (IMC). Las intervenciones dietéticas intensivas pueden ser seguras, con un beneficio modesto a corto plazo de importancia clínica incierta. No se identificaron pruebas directas de los beneficios o daños del examen o tratamiento infantil en los resultados en la edad adulta.
Fuentes de financiamiento: Esta investigación fue financiada por AHRQ (Agency for Healthcare Research and Quality) bajo un contrato para apoyar el USPSTF.

Conflictos de interés: no reportados. 
Tabla 2. Niveles de lípidos plasmáticos en niños y adolescentes

\begin{tabular}{|c|c|c|c|c|}
\hline \multicolumn{2}{|c|}{ Categoria } & Aceptable (mg/dl) & Limite (mg/dl) & $\begin{array}{c}\text { Alto o Bajo (para HDL-c y Apo } \\
\text { A-1) (mg/dl) }\end{array}$ \\
\hline \multicolumn{2}{|l|}{ Colesterol total } & $<170$ & $170-199$ & $\geq 200$ \\
\hline \multicolumn{2}{|c|}{ LDL-C } & $<100$ & $110-129$ & $\geq 130$ \\
\hline \multicolumn{2}{|l|}{ Apo B } & $<90$ & $90-109$ & $\geq 110$ \\
\hline \multirow[t]{2}{*}{ Triglicéridos } & 0-9 años & $<75$ & $75-99$ & $\geq 100$ \\
\hline & 10-19 años & $<90$ & $90-129$ & $\geq 130$ \\
\hline \multicolumn{2}{|l|}{ HDL-C } & $>45$ & $40-45$ & $\leq 40$ \\
\hline \multicolumn{2}{|l|}{ Apo A-1 } & $>120$ & $115-120$ & $\leq 115$ \\
\hline
\end{tabular}

\section{Comentario}

La hipercolesterolemia multifactorial o poligénica es de etiología desconocida. Se infiere que ocurre por alteraciones en diversos genes reguladores del metabolismo del colesterol que, en combinación con factores ambientales, especialmente la dieta, darían lugar al aumento del colesterol. Es la forma más frecuente de hipercolesterolemia primaria en la población general, con una prevalencia estimada del 5 al $20 \%$. La dislipidemia no es una enfermedad sino un factor de riesgo para aterosclerosis y enfermedad cardíaca en edad adulta ${ }^{1}$.

Se define hipercolesterolemia cuando los valores de colesterol total son mayores a $200 \mathrm{mg} / \mathrm{dl}$ o de LDL mayores a $130 \mathrm{mg} / \mathrm{dl}$ (ver Tabla 2) ${ }^{2}$.

El rastreo, en este grupo etario, podría tener el beneficio potencial de identificar a los jóvenes afectados, disminuir los valores de colesterol a largo plazo para prevenir o retrasar los eventos cardiovasculares en la edad adulta. Sin embargo, la determinación de lípidos en la niñez y adolescencia no identifica adecuadamente a los adultos con dislipidemia y no se ha podido establecer una clara correlación entre valores elevados de colesterol en la niñez o adolescencia y la incidencia futura de enfermedad coronaria y cerebrovascular. El rastreo de hipercolesterolemia en niños o adolescentes sigue siendo un tema controversial, ya que además de lo mencionado, al analizar la evidencia disponible, quedan varios interrogantes aún sin responder. Por ejemplo, ¿a quién y cada cuánto rastrear? ¿Qué determinaciones utilizar para rastreo? Por otro lado, no hay evidencia del riesgo de un tratamiento hipolipemiante a largo plazo iniciado a edades tempranas ${ }^{3-5}$. En coincidencia con la recomendación emitida por la USPSTF, las últimas guías de AAP, NCEP, CTFPHC no recomiendan el rastreo universal, sino, en poblaciones consideradas de riesgo: historia familiar de enfermedad cardiovascular prematura en padres, abuelos o tíos (varones $<55$ años o mujeres $<65$ años) o padres con dislipemia conocida o CT mayor o igual a $240 \mathrm{mg} / \mathrm{dl}$; factores de alto riesgo: hipertensión arterial que requiere tratamiento farmacológico ( presión arterial > percentilo $99+5 \mathrm{mmHg}$ ), tabaquismo, IMC $\geq$ percentilo 97 , diabetes mellitus, enfermedad renal crónica, trasplante de órganos; factores de moderado riesgo: hipertensión arterial que no requiere tratamiento farmacológico, percentilo de IMC $>95$ y $<97$, HDL-C $<40 \mathrm{mg} / \mathrm{dl}$, enfermedad inflamatoria crónica (lupus eritematoso sistémico, artritis idiopática juvenil), VIH y síndrome nefrótico.

\section{Conclusiones del comentador}

La evidencia actual es insuficiente para evaluar el equilibrio de los beneficios y los daños del rastreo de hipercolesterolemia en NyA menores de 20 años.

No hay evidencia directa sobre el beneficio de la detección temprana, el tratamiento y la incidencia de eventos cardiovasculares en la edad adulta, ni de los daños relacionados con el rastreo (como sobrediagnóstico, etiquetamiento) y la seguridad del tratamiento a largo plazo con estatinas.

Son necesarios estudios, idealmente ECCA, con alta calidad metodológica y con mayor tiempo de seguimiento que evalúen la costo-efectividad de los distintos métodos de rastreo y la eficacia/seguridad de suplementos alimentarios y medicación para el tratamiento de NyA con hipercolesterolemia

\section{David Colica [ Servicio de Medicina Familiar y Comunitaria. Hospital Italiano de Buenos Aires. david.colica@ hospitalitaliano.org.ar ]}

Colica D. Rastreo de hipercolesterolemia en niños y adolescentes. Evid Actual Pract Ambul 2018;21(4):102-103. Comentado de: Lozano P, y col. Lipid Screening in Childhood and Adolescence for Detection of Multifactorial Dyslipidemia: Evidence Report and Systematic Review for the US Preventive Services Task Force. JAMA. 2016;316(6):634-644. PMID: 27532918.

\section{Referencia}

1. M.T. Muñoz Calvo. Dislipemias. Pediatria Integral 2015; XIX (5): 355-364

2. Daniels SR, Benuck I, Christakis DA, et al. Expert panel on integrated guidelines for cardiovascular health and risk reduction in children and adolescents: Full report, 2011. National Heart Lung and Blood Institute. Disponible en: http://www.nhlbi.nih.gov/guidelines/cvd_ped/peds_guidelines_full.pdf.

3. National Cholesterol Education Program. Report of the expert panel on blood cholesterol levels in children and adolescents. Pediatrics. $1992 ; 89$ (Suppl.) 525-84. 4. Expert Panel on Integrated Guidelines for Cardiovascular Health and Risk Reduction in Children and Adolescents; National Heart, Lung, and Blood Institute..Pediatrics. 2011;128(suppl 5):213-256.

5. Newman TB, y col. Lipid Screening in Children: Low-Value Care. JAMA Intern Med. 2016;176(10):1437-1438 